\title{
Understanding the growth mechanism of hematite nanoparticles: The role of maghemite as an intermediate phase
}

\author{
Suresh Bandi, Ajeet K. Srivastav* \\ Department of Metallurgical and Materials Engineering, Visvesvaraya National Institute of \\ Technology, Nagpur - 440 010, India
}

\section{S.1: Synthesis protocol of chemical co-precipitation}

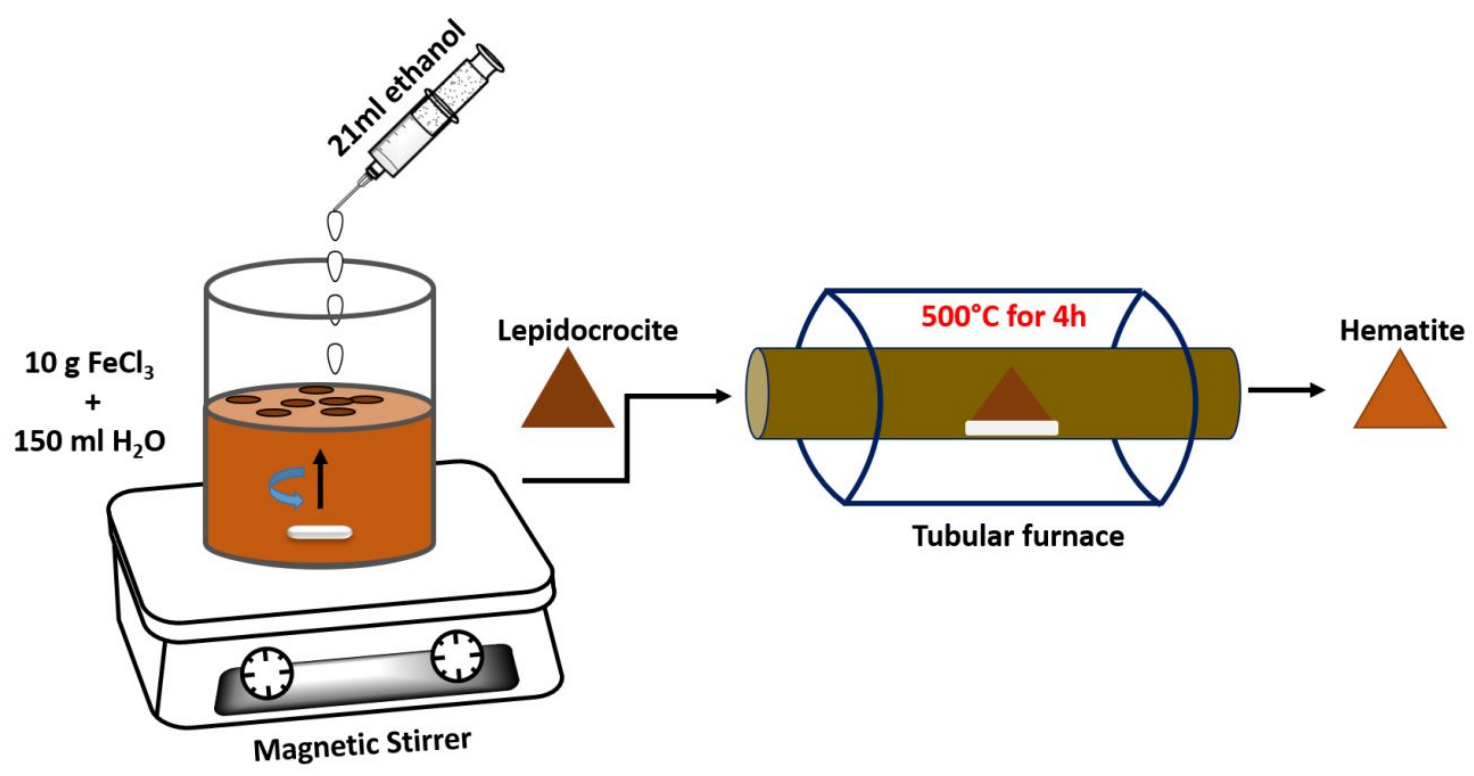

Fig. S1 The schematic representation of the chemical co-precipitation method and subsequent heat treatment.

\section{S.2: The crystallographic pathways of lepidocrocite to maghemite.}

The formation of hematite $\left(\alpha-\mathrm{Fe}_{2} \mathrm{O}_{3}\right)$ follows two steps: 1) precipitation of ferrihydrite/iron oxyhydroxide, i.e., lepidocrocite $(\gamma-\mathrm{FeOOH})$, and 2$)$ dehydration of iron oxyhydroxide to hematite via calcination ${ }^{1}$. The initial phase formation (i.e., lepidocrocite) purely depends on the experimental conditions and precursors employed for the synthesis. Therefore, the initial

\footnotetext{
${ }^{*}$ Corresponding author

E-mail: srivastav.ajeet.kumar@gmail.com
} 
predominant phase directs the whole pathway of hematite. The transformation of lepidocrocite to hematite crosses through the intermediate phase of maghemite $\left(\gamma-\mathrm{Fe}_{2} \mathrm{O}_{3}\right)^{2,3}$.

The first step involves the desorption of physisorbed $\mathrm{H}_{2} \mathrm{O}$ molecules and dehydroxilization. The lateral transformation results in hematite at temperatures above $350^{\circ} \mathrm{C}$. Also, there involves a crystallographic orientation relation between these phases.

The lepidocrocite is a layered compound that possesses orthorhombic crystal symmetry with $\mathrm{cmcm}$ space group. The structure built by the double layer of octahedron connected by edge-sharing is parallel to $a \& c$ and perpendicular to the $b$ axis. These layers are bonded together with the help of the zigzag patterned hydroxyl network. The anions $\left(\mathrm{O}^{2-}\right.$ or $^{\left.-\mathrm{OH}^{-}\right)}$of this network follow a CCP (cubic close-packed) framework, where the layers are stacked in [150] direction, which corresponds to the [111] direction in the distorted cubic cell. During the dehydration treatment, the hydrogen atoms migrate to form $\mathrm{H}_{2} \mathrm{O}$, and immediate desorption occurs through evaporation. This process strips hydroxyl groups and few oxygen atoms as well. This dehydroxilization may cost the one-fourth removal of oxygen atoms or half of the oxygen atoms from the surface as the interior remains intact. ${ }^{3}$ Subsequently, it turns to the cubic oxygen network with appropriate layer amalgamation, i.e., maghemite of the inverse spinal structure. It consists of the corner and edge-sharing octahedral of $\left(\mathrm{FeO}_{6}\right)$ arranged in the CCP framework. ${ }^{4}$ During this transformation, the (100) and [001] of $\gamma$-FeOOH changes to (110) and $[1 \overline{10}]$ of $\gamma-\mathrm{Fe}_{2} \mathrm{O}_{3}{ }^{5}$ The schematic representation of such topotactical transformation with the orientation relationship $(100)_{L} / /(110)_{M}$ is shown in fig.S2.

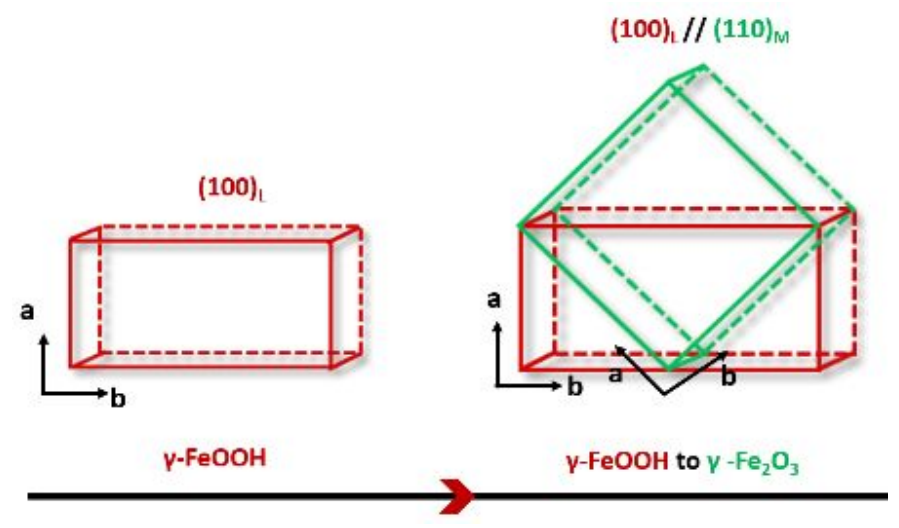

Fig. S2 The crystallographic pathway of lepidocrocite $\rightarrow$ maghemite. The (100) plane of the orthorhombic $\gamma$-FeOOH is in parallel to the $(110)_{M}$ plane of CCP $\gamma-\mathrm{Fe}_{2} \mathrm{O}_{3}$. 


\section{References:}

(1) Zhang, W.-J.; Huo, C.-F.; Feng, G.; Li, Y.-W.; Wang, J.; Jiao, H. Dehydration of Goethite to Hematite from Molecular Dynamics Simulation. J. Mol. Struct. THEOCHEM 2010, 950 (1-3), 20-26.

(2) Morris, R. V.; Golden, D. C.; Shelfer, T. D.; Lauer, H. V. Lepidocrocite to Maghemite to Hematite: A Pathway to Magnetic and Hematitic Martian Soil. Meteorit. Planet. Sci. 1998, 33 (4), 743-751.

(3) Gendler, T. S.; Shcherbakov, V. P.; Dekkers, M. J.; Gapeev, A. K.; Gribov, S. K.; McClelland, E. The Lepidocrocite-Maghemite-Haematite Reaction Chain-I. Acquisition of Chemical Remanent Magnetization by Maghemite, Its Magnetic Properties and Thermal Stability. Geophys. J. Int. 2005, 160 (3), 815-832.

(4) Giovanoli, R.; Brütsch, R. Kinetics and Mechanism of the Dehydration of $\gamma-\mathrm{FeOOH}$. Thermochim. Acta 1975, 13 (1), 15-36.

(5) Takada, T.; Kiyama, M.; Shimizu, S. Morphological and Crystallographic Studies on the Oriented Transfomation in $\gamma-\mathrm{FeOOH}$ and Its Decomposed Oxides. Bull. Inst. Chem. Res. Kyoto Univ. 1965, 42 (6), 505-510. 